\title{
Editorial
}

\section{SFCO and the storm of COVID-19}

\author{
Jacques Henri Torres*
}

President of the SFCO

(Received: 15 July 2020, accepted: 15 July 2020)

A major sanitary event took place in 2020: the worldwide spread of a new coronavirus called "severe acute respiratory syndrome coronavirus 2" (SARS-CoV-2), agent of the worldwide pandemic of coronavirus disease 2019 (COVID-19). It seems that the virus reached Europe in December 2019 or January 2020. The first death of a patient due to COVID-19 out of China took place in Paris in February 2020. In France, the disease mainly affected the Paris Region and the East of the country. A 1-week meeting of the Christian Open-Door Church held in February in Mulhouse (East of France) played a key role in the spreading of the disease: the participants carried the virus back home into many French regions. In the Paris Region, overcrowding and specially promiscuity in the public transportation are thought to have been a major factor in the virus transmission.

SARS-CoV-2 can remain infectious on surfaces, from several hours up to several days depending on the material. However, the major means of transmission is the inhalation of small droplets produced by an infected person talking, sneezing or coughing. Therefore, social distancing is of crucial importance to reduce the spread of the pandemic. It was the very first time in the history of mankind that distancing measures were applied on the global level. In France, a total lockdown of the population was ordered by the government from March $17^{\text {th }}$ to May $11^{\text {th }} 2020$. After that, social distancing and the wearing of face masks remained mandatory in many situations, especially public transportation and health institutions.

COVID-19 is a severe illness: over $2 \%$ of the diagnosed cases died because there was no effective therapy by the time this paper was written, and no vaccine available either. Besides its own medical impact, COVID-19 was also a worldwide economic disaster because social distancing and lockdown terribly affected the economic life of billions. Other consequences were quite unexpected, such as a drastically

\footnotetext{
*Correspondence: jacques-henri.torres@umontpellier.fr
}

decreased pollution due to the stopping of factories and road traffic. Likewise, a spectacular decline in road traffic accident injuries was noted in the hospitals. On the other hand, unfortunately, stress-related illnesses increased, and so did domestic accidents and domestic violence. These facts changed the daily practise of oral surgeons.

As SARS-CoV-2 is transmitted by saliva and respiratory droplets, the oral care practitioners are particularly exposed to contamination. In France, as well as the dental offices, most of the private practice oral surgery offices have been closed during the lockdown. As a result of this situation, a serious concern rose about the management of dental emergencies. Patients with acute pain, severe infection or alarming mouth lesions could not find a place to be taken care of. This occurred to be an unprecedent case of public health service failure in recent memory in an industrialized nation. Of course, some centers remained open in hospitals and also in private offices, thanks to the Dental National Council. But they were very few and could only manage a very small number of patients because of very stringent conditions of practice. Indeed, the preventive measures (temperature-taking, questioning for COVID-19 symptoms at the welcome desk, handwashing or friction with hydroalcoholic solution, distancing in the waiting room, antivirus mouth rinse) significantly increased the time of the patient reception and preparation. In the treatment rooms, oral surgeons and nurses had to wear specific personal protective equipments, including notably protective eyewear and specific masks, much less comfortable than the usual ones. One of the main issues have been a drastic restriction of the access to general anaesthesia surgery, as a result of the mandatory changes of hospitals organization because of COVID, and a severe shortage of anaesthetic drugs.

Compared to many other surgery specialities, oral surgery has a much higher risk for COVID-19 droplet contamination because of saliva aerosol creation by micro motor handpieces and turbines.

Across the time of the lockdown, the French Society of Oral Surgery (SFCO) contributed to the conception of guidelines to 
help dentists and oral surgeons in their practice. SFCO also produced specific recommendations to clarify the question of the use of corticosteroid therapy during the pandemic.

As a result of this challenging period, everyone was tired. Not only did the preventive measures took time but they also increased the stress for both the patient and the practitioner. A major consequence was a substantial loss of chance as a consequence of delayed diagnosis and restricted access to surgery. After the end of the lockdown, many cancer cases were seen at a more advanced stage than usual.

All in all, we lived a very exceptional situation at a professional level. And also at a personal level since many of us, or many members of our families and entourage unfortunately caught the disease.

I guess all the oral surgeons did not feel they were heroes, but they probably were. 